\title{
Preemptive Morphine Analgesia Attenuates the Long-Term Consequences of Neonatal Inflammation in Male and Female Rats
}

\author{
JAMIE L. LAPRAIRIE, MALCOLM E. JOHNS, AND ANNE Z. MURPHY \\ Department of Biology, Georgia State University, Atlanta, Georgia 30303-3088
}

\begin{abstract}
Despite mounting evidence on the importance of pain management in preterm infants, clinical use of analgesics in this population is limited. Our previous studies have shown that neonatal inflammation results in long-term alterations in adult somatosensory thresholds, characterized by decreased baseline nociceptive sensitivity, and enhanced hyperalgesia after a subsequent inflammatory insult. The present studies were conducted to determine whether preemptive morphine attenuates these negative consequences. At P0, pups received an injection of morphine sulfate before an intraplantar injection of $1 \%$ carrageenan. Control pups received either saline (SAL) followed by intraplantar carrageenan, morphine sulfate followed by intraplantar SAL, or SAL followed by intraplantar SAL. Preemptive morphine significantly attenuated neonatal injuryinduced hypoalgesia in adolescence and adulthood. Similarly, morphine pretreated animals displayed significantly less hyperalgesia and recovered faster from a subsequent inflammatory insult compared with controls. Neonatal morphine had no significant effect on morphine analgesia in adulthood. Interestingly, neonatally injured animals that did not receive morphine displayed a significant rightward shift in the morphine dose-response cuve in the absence of peripheral inflammation. Together, these results demonstrate that preemptive morphine significantly attenuates the long-term behavioral impact of neonatal inflammatory injury. (Pediatr Res 64: 625-630, 2008)
\end{abstract}

$\mathrm{U}$ ntil the late 1980s, many in the medical community believed that neonates were incapable of experiencing pain (1). It is now well established, however, that premature infants are highly responsive to noxious stimulation $(2,3)$ and generate developmentally specific and distinct responses to noxious stimuli $(4,5)$. Nociceptive responses to noxious stimulation have been demonstrated in preterm neonates using an array of physiologic, biochemical, and behavioral measures $(6,7)$. Cortical activation has also been reported in response to noxious stimulation in preterm neonates at $25 \mathrm{wk}$, suggesting the potential for higher level processing of pain $(8,9)$.

Accumulating evidence indicates that exposure to invasive procedures during the neonatal period leads to both short- and long-term alterations in nociceptive processing (10-13). For example, a higher frequency of invasive procedures in preterm infants has been associated with dampened pain responses at

Received May 30, 2008; accepted July 12, 2008.

Correspondence: Anne Z. Murphy, Ph.D., Department of Biology, Center for Behavioral Neuroscience, Georgia State University, 24 Peachtree Center Ave, 402 Kell Hall, Atlanta, GA 30303-3088; e-mail: amurphy@gsu.edu

This work was supported by National Institute of Health grants DA16272 and AR49555 awarded to Anne Z. Murphy, the Center for Behavioral Neuroscience NSF: IBN 9876754, and the Georgia State University Brains and Behavior Program.
32 wk of age (14). Decreased facial responsiveness to immunization at 4 and 8 mo (15), and blunted pain sensitivity has also been reported in former preterm neonates (10).

Animal studies have also reported that neonatal injury induces long-term alterations in somatic and visceral sensitivity $(12,13,16)$. In particular, animals that received intraplantar carrageenan (CGN) at birth display significant hypoalgesia in the previously injured and uninjured paws in adulthood $(12,13)$. Similar changes in nociceptive sensitivity have also been reported using intraplantar formalin (17). Neonatal injury also results in enhanced hyperalgesia after a subsequent insult in adulthood and significantly decreases the rate of recovery $(12,13)$.

The use of opioid analgesics in the treatment of neonatal pain is widely debated. Accumulating data, however, suggest that premature infants undergoing invasive procedures benefit from the use of opioid analgesics $(18,19)$. For example, altered pain responses in former preterm neonates can be predicted by the number of previous painful procedures and are normalized by the use of morphine (14). Moreover, postoperative morphine in preterm and full-term infants reduces behavioral and hormonal stress responses $(20,21)$, and is associated with decreased mortality (18).

There is evidence that opioid analgesics are efficacious in neonatal rodents (22). Few studies, however, have examined whether opioid analgesics can be used to prevent the consequences of neonatal injury (23). Therefore, the present studies were conducted to determine whether neonatal morphine 1) attenuates neonatal injury-induced hypoalgesia in adulthood; 2) reduces enhanced hyperalgesia observed after adult inflammation and impacts the rate of recovery; and 3) alters the response to morphine in adulthood.

\section{METHODS}

Animals. Time-pregnant Sprague-Dawley rats were obtained on the 14th day of gestation (E14) (Zivic Miller) and housed individually. Animals were maintained on a 12:12 h light:dark cycle, with food and water available ad libitum. Offspring from the litters were combined on the day of birth, divided up equally, and randomly assigned to a neonatal treatment condition and dam. All litters were reared identically, weaned at P21, and housed with same-sex littermates in groups of 2-3. All experiments were approved by the Georgia State University Animal

Abbreviations: CFA, Complete Freund's adjuvant; CGN, carrageenan; MDS, mean difference score; MOR, mu opioid receptor; MOR-LI, mu opioid receptor-like immunoreactivity; MS, morphine sulfate; $\mathbf{P}$, postnatal day; PWL, paw withdrawal latency; SAL, saline 
Care and Use Committee and were conducted in compliance with the guidelines established by the International Association for the Study of Pain.

Early life manipulations. On the day of birth ( $\mathrm{P} 0)$, male and female rat pups received MS (MS; $2 \mathrm{mg} / \mathrm{kg}$, i.p.) or SAL $15 \mathrm{~min}$ before a unilateral intraplantar injection of $1 \%$ CGN (5 $\mu \mathrm{L})$ or SAL. This dose of morphine was chosen based on previous studies $(22,24)$. In preliminary studies, we noted that paw inflammation peaked at $5 \mathrm{~h}$ post-CGN. Therefore, at $5 \mathrm{~h}$ post-CGN or SAL, a second dose of either MS or SAL was administered. This resulted in a total of four groups: MS + SAL, MS+CGN, SAL+CGN, and SAL+SAL. All pups within a litter received the same neonatal treatment.

Maternal behavior. Maternal behavior was observed daily at 18:00 $\mathrm{h}$ for 60 min from P0-P7. Maternal observations were conducted by both direct observations and by videotape for later offline analysis. Specific maternal behaviors were recorded including pup licking/grooming, nursing posture (crouching), pup retrieval, nest construction, eating/drinking, exploring, inactive/napping, and self-grooming. Observations were conducted by an individual blind to the neonatal group assignment.

Baseline nociceptive behavior. On postnatal days 40 and 60 (P40 and P60), baseline paw withdrawal (PWL) and tail flick (TFL) latencies in response to noxious thermal and mechanical stimuli were determined. Thermal testing was conducted using the Paw Thermal Stimulator (UCSD, CA). In this test, animals were placed in a clear plastic testing chamber on a glass surface and allowed to acclimate for $30 \mathrm{~min}$ before testing. A radiant beam of light beneath the glass base was directed at the plantar surface of the each hindpaw or 1.5 inches from the distal end of the tail, and the withdrawal latency was electronically measured (25). To avoid potential tissue damage, a 20 -s automatic termination of the heat stimulus was imposed if withdrawal did not occur. Mechanical testing was conducted using a Dynamic Plantar Aesthesiometer (Ugo Basile, Italy). Animals were placed in Plexiglas chambers above a wire mesh, and allowed to acclimate for $30 \mathrm{~min}$ before testing. A metal probe was directed at the hind paw and the force applied electronically increased until PWL occurred. Both time and force were recorded, with an automatic cutoff of $60 \mathrm{~s}$ and $50 \mathrm{~g}$, respectively. Male and female rats were tested separately. For both tests, the average response of three trials was taken; all trials were separated by a 5-min intertrial interval.

Nociceptive behavior after re-inflammation. After baseline PWL determination at P60, animals received an intraplantar injection of Complete Freund's adjuvant (CFA; 1:1 CFA: SAL solution; $200 \mu \mathrm{L}$; Sigma Chemical Co.) into the neonatally injured hindpaw. CFA was used for re-injury as neonatally injured animals may potentially develop antibodies against CGN, thereby limiting its potency for use as an inflammatory agent. Twenty four hours after CFA, paw diameter and PWLs were tested as aforementioned.

Recovery after re-inflammation. Paw diameter and PWLs were tested at 7 , 14 , and $21 \mathrm{~d}$ after CFA re-inflammation (P60) to determine the impact of morphine pretreatment on the response to recovery from a subsequent insult in adulthood.

Response to morphine in adulthood after neonatal morphine exposure. The effect of neonatal morphine on adult morphine responses was tested in the absence of peripheral inflammation. After baseline PWL determination, animals were administered increasing cumulative doses of morphine $(1.8,1.4,2.4,2.4$, 2.0 , and $8.0 \mathrm{mg} / \mathrm{kg}$ s.c. resulting in quarter $\log$ doses of $1.8,3.2,5.6,8.0,10.0$, and $18.0 \mathrm{mg} / \mathrm{kg}$; Sigma Chemical Co.) (26). PWLs were determined $15 \mathrm{~min}$ after each injection, followed by administration of the subsequent injection 5 min later.

Mu opioid receptor western blot analysis. Western blot analysis was conducted to confirm that mu opioid receptors (MOR) are present centrally and peripherally on P0. Five hours after birth, rat pups were rapidly decapitated and brain, spinal cord, and paw tissue were removed and collected on dry ice. Dams were decapitated and maternal blood samples taken. Tissue and blood were stored at $-80^{\circ}$. Tissue samples were homogenized in Homogenization Buffer (50 mM HEPES pH 7.4, $1 \mathrm{mM}$ EDTA, and 0.001\% Protease Inhibitor Cocktail; Sigma Chemical Co., USA) on ice. The samples underwent freeze/thawing three times on dry ice and $37^{\circ} \mathrm{C}$ waterbath, respectively, and were stored at $-80^{\circ} \mathrm{C}$. Sample protein concentrations were determined with a BCA Protein Assay Kit (Pierce) against a BSA standard curve. Next, $20 \mu \mathrm{g}$ of each sample was run on $12 \%$ acrylamide gels, and transferred to polyvinylidene fluoride (PVDF) membranes (Bio-Rad). PVDF membranes were blocked in $5 \%$ nonfat dry milk in TTBS $(0.1 \%$ Tween in $20 \mathrm{mM}$ Tris- $\mathrm{HCl} ; \mathrm{pH} 7.5,175 \mathrm{mM} \mathrm{NaCl}$ ) overnight at $4^{\circ} \mathrm{C}$. PVDF membranes were washed three times with TTBS, 10 min each, then incubated with rabbit anti-MOR (1:50,000; Sigma Chemical Co.) and rabbit anti-GAPDH $\left(1: 300,000\right.$; Covance) in $2 \%$ nonfat dry milk in TTBS overnight at $4{ }^{\circ} \mathrm{C}$. Membranes were washed three times with TTBS, $10 \mathrm{~min}$ each, and incubated with anti-rabbit HRP conjugated antibodies (1:3000; Cell Signaling) for $1 \mathrm{~h}$. Membranes were subsequently washed with TTBS three times, 10 min each, and washed in TBS $(20 \mathrm{mM}$ Tris- $\mathrm{HCl} \mathrm{pH} 7.5,175 \mathrm{mM} \mathrm{NaCl})$ for $30 \mathrm{~min}$. Bands were visualized via chemiluminescence using LumiGLO (KPL). To confirm that the MOR protein observed in the tissue samples was not the result of mu receptors located on maternal immune cells, western blot analysis was also conducted on maternal blood samples.

Statistical analysis. Data are expressed as either raw withdrawal latencies or mean difference scores (MDS). All values are reported as Mean \pm SEM. Data were analyzed for significant main effects of neonatal treatment and sex using ANOVA and repeated-measures ANOVA was used to analyze recovery postCFA data; $p<0.05$ was considered statistically significant. A priori specified post hoc tests were conducted using the method of Sheffe as warranted to determine significant mean differences. Where multiple comparisons were made, $p$ values were adjusted accordingly using the Bonferroni method. Morphine dose-response curves were plotted and the half maximal effective doses (ED50) were calculated using percent maximal possible effect (\%MPE) data, defined as [(Dose mg/kg-baseline PWL)/(20.0-baseline PWL)]100 (GraphPad, Prism). Analysis of variance was used to compare differences between ED50 values.

\section{RESULTS}

Neonatal morphine attenuates hypoalgesia in adulthood. Preemptive morphine significantly attenuated both thermal (Fig. 1) and mechanical (Fig. 2) hypoalgesia induced by neonatal inflammation at P60. Animals that received $\mathrm{SAL}+\mathrm{CGN}$ displayed significant thermal hypoalgesia in both the injured and uninjured paws, with females displaying PWLs of approximately $14 \mathrm{~s}$ and males $11.5 \mathrm{~s}(p<0.05$ treatment; $p<0.05 \mathrm{sex})$, versus PWLs of $10 \mathrm{~s}$ in SAL $+\mathrm{SAL}$ animals. In contrast, morphine pretreated animals $(\mathrm{MS}+\mathrm{CGN})$ were not significantly different from uninjured controls. Consistent with our previous study (13), a significant
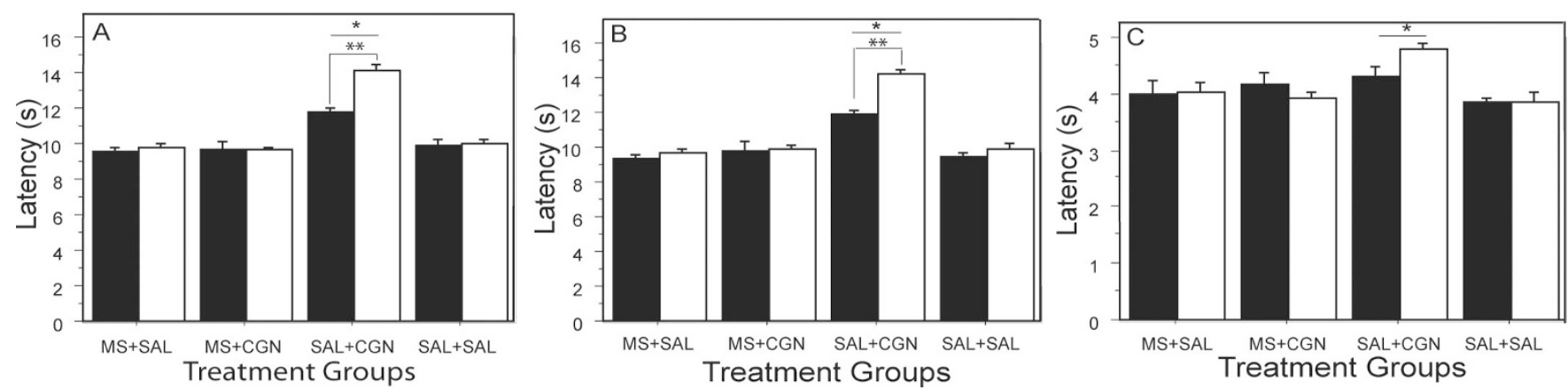

Figure 1. Morphine attenuates thermal hypoalgesia in adulthood. MS + CGN animals displayed significantly shorter PWLs compared with SAL + CGN in response to thermal stimulation applied to the $(A)$ uninjured paw $[F(3,73)=61.330, p<0.0001] ;(B)$ injured paw $[F(3,73)=80.066, p<0.0001]$. $(C)$ MS $+C G N$ animals displayed significantly reduced TFLs compared with SAL +CGN animals $[F(3,73)=5.879, p=0.0012]$. Neonatally injured females displayed significantly longer PWLs compared with males; uninjured paw $[F(1,73)=8.912, p=0.0039]$ and injured paw $[F(1,73)=16.914, p<0.0001] . n=5-15$ rats per group/per sex; *significant main effect of treatment; **significant main effect of sex; male: $\mathbf{\square}$; female: $\square$. 

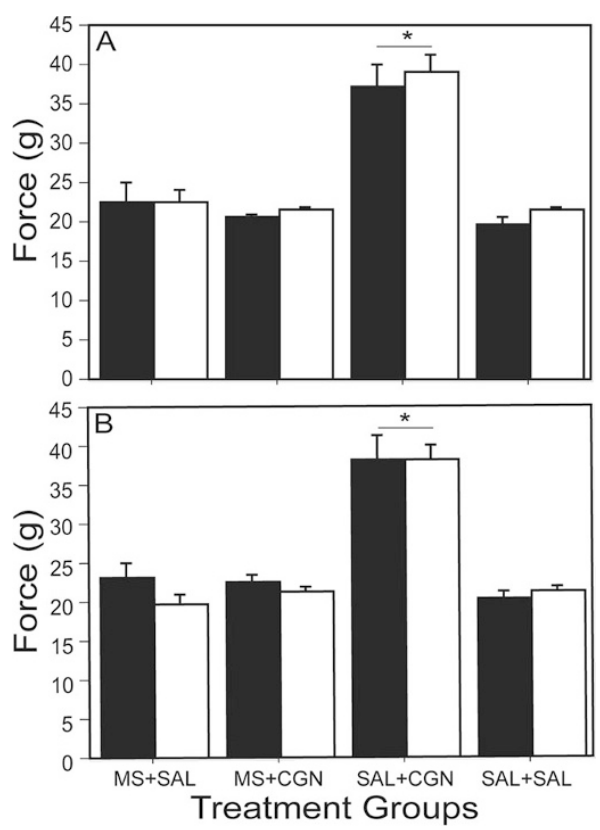

Figure 2. Morphine attenuates mechanical hypoalgesia in adulthood. MS+CGN displayed significantly reduced force $(g)$ to withdrawal compared with $\mathrm{SAL}+\mathrm{CGN}$ in response to mechanical stimulation applied to the $(A)$ uninjured paw $[F(3,40)=50.604, p<0.0001] ;(B)$ injured paw $[F(3,40)=$ $56.898, p<0.0001] . n=5-15$ rats per group/per sex; *significant main effect of treatment; male: $\mathbf{\square}$; female: $\square$.

main effect of sex on injury-induced thermal hypoalgesia was noted (Fig. $1 A$ and $B$ ). Morphine also reduced the injuryinduced hypoalgesia present in the tail at P60 (Fig. 1C) $(p<$ $0.05)$. Furthermore, a significant reduction in the mechanical force threshold was present in morphine pretreated animals in both paws in adulthood (P60) (Fig. $2 A$ and $B)(p<0.05)$. Significant morphine attenuation of the inflammation-induced hypoalgesia was also observed at P40 (data not shown).

Neonatal morphine alters the response to re-injury and recovery. Neonatal morphine significantly attenuated CFAinduced hyperalgesia at $24 \mathrm{~h}$ compared with controls (Fig. 3A) $(p<0.05)$. At 24 h post-CFA, PWLs for SAL + CGN animals
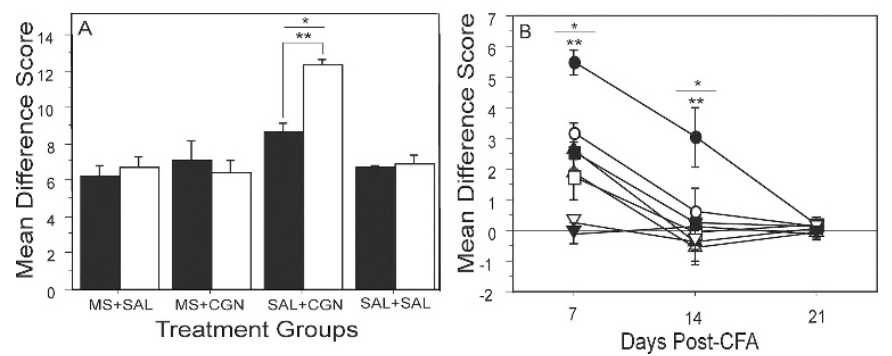

Figure 3. Morphine attenuates the hyperalgesia after re-inflammation and increases the rate of recovery. MS+CGN animals displayed significantly lower MDS (i.e., reduced hyperalgesia) compared with SAL+CGN animals (A) 24 -h post-CFA $[F(3,36)=22.524, p<0.0001]$; male: $\square$; female: $\square$. (B) At 7, 14 and $21 \mathrm{~d}$ post-CFA, SAL+CGN animals displayed a significantly higher MDS (i.e., reduced rate of recovery) compared with controls $[F(6,76)=10.644, p<0.0001]$. Females displayed significantly higher MDS (i.e., recovered slower) compared with males $[F(2,76)=3.240, p<0.05]$. $n=5-7$ rats per group/per sex; *significant main effect of treatment; **significant main effect of sex; male: $\mathbf{\square}$; female: $\square$ (MS +SAL- $\Delta$; MS+CGN- $\square$; SAL + CGN-O; SAL +SAL- $\nabla$ ). were approximately $2 \mathrm{~s}$ in females (MDS, of $12 \mathrm{~s}$ ), and approximately $3.5 \mathrm{~s}$ in males (MDS $8.5 \mathrm{~s}$ ). Neonatally injured females displayed significantly enhanced thermal hyperalgesia (i.e., greater MDS) at $24 \mathrm{~h}$ after intraplantar CFA compared with males as previously reported ( $p<0.05$; Fig. 3A) (13). The rate of recovery was also significantly reduced in morphine pretreated compared with control animals (Fig. 3B). $\mathrm{SAL}+\mathrm{CGN}$ animals continued to show signs of hyperalgesia at $14 \mathrm{~d}(p<0.05$; MDS of $1 \mathrm{~s}$ in males and $3 \mathrm{~s}$ in females). In contrast, MS + CGN animals had completely recovered at this time point (MDS of 0 ), similar to control animals ( $p<$ 0.05 ). Furthermore, a significantly decreased rate of recovery was noted in injured females compared with injured males; where females displayed greater MDS at 7 and $14 \mathrm{~d}$ post-CFA $(p<0.05)$.

Neonatal morphine does not affect morphine analgesia in adulthood. Neonatal morphine did not significantly alter morphine's antinociceptive effects in adulthood in males or females (Fig. $4 A$ and $B$ ). As summarized in Table 1, morphine pretreated females (MS+CGN) displayed similar ED50 values as morphine (MS+SAL) and saline (SAL+SAL) control females. Interestingly, a significant shift in ED50 was noted in neonatally injured females $(\mathrm{SAL}+\mathrm{CGN})$ that did not receive morphine $(p<0.05)$. Similarly, neonatal morphine in injured males did not alter adult morphine responses (Fig. 4B). Nevertheless, SAL +CGN males also displayed a significant shift in ED50 compared with MS+CGN and control males. Finally, females had significantly higher ED50 values compared with males ( $p<0.05$; Table 1$)$, as previously reported (27).

Mu opioid receptors are present at birth. As shown in Figure 5, MOR-like immunoreactivity (LI) is present on P0 in the brain, spinal cord, and right hindpaw tissue. To control

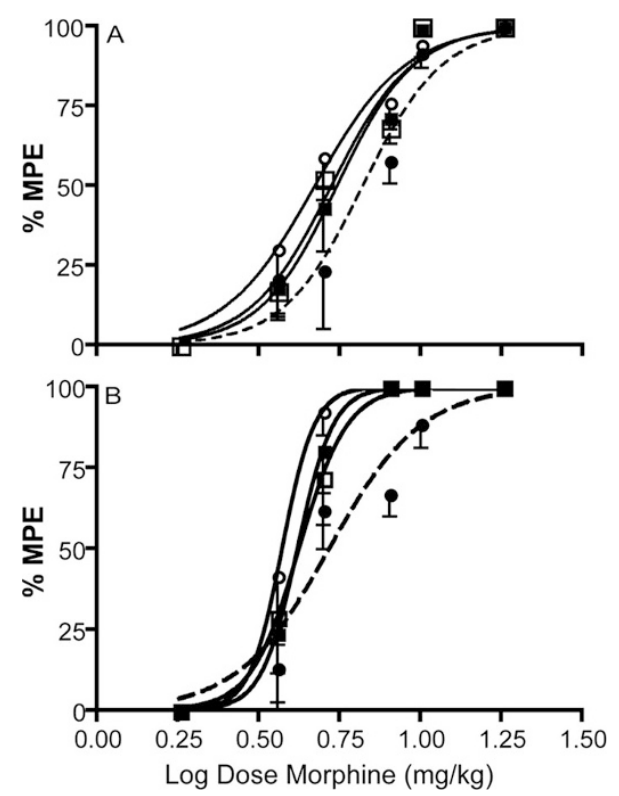

Figure 4. Neonatal morphine has no effect on morphine analgesia. Neonatal morphine had no effect on morphine potency in $(A)$ females; or $(B)$ males in adulthood. Neonatal injury (SAL +CGN) (- - ) produced a significant shift in the morphine dose-response curve in $(A)$ females $[F(3,250)=2.982, p=$ 0.03]; and $(B)$ males $[F(3,208)=2.979, p=0.03]$ compared with control animals (-). $n=6-13$ rats per group/per sex; MS + CGN- $\square$; SAL+SAL-O; $\mathrm{MS}+\mathrm{SAL}-\square ; \mathrm{SAL}+\mathrm{CGN}-\mathrm{O}$ 
Table 1. Morphine ED50 values

\begin{tabular}{lcccc}
\hline Treatment & Female & $95 \%$ C.I. & Male & $95 \%$ C.I. \\
\hline MS+/CGN & 5.49 & $4.82-6.26$ & $4.17 *$ & $3.70-4.71$ \\
SAL + SAL & 4.69 & $4.16-5.29$ & $3.74^{*}$ & $3.41-4.11$ \\
MS+SAL & 5.28 & $4.77-5.84$ & $4.22^{*}$ & $3.76-4.74$ \\
SAL + CGN & $6.60 \dagger$ & $5.54-7.86$ & $5.24 * \dagger$ & $4.35-6.32$ \\
\hline
\end{tabular}

$* p<0.05$ significant effect of sex.

$\dagger p<0.05$ significant effect of treatment.

C.I., confidence interval.

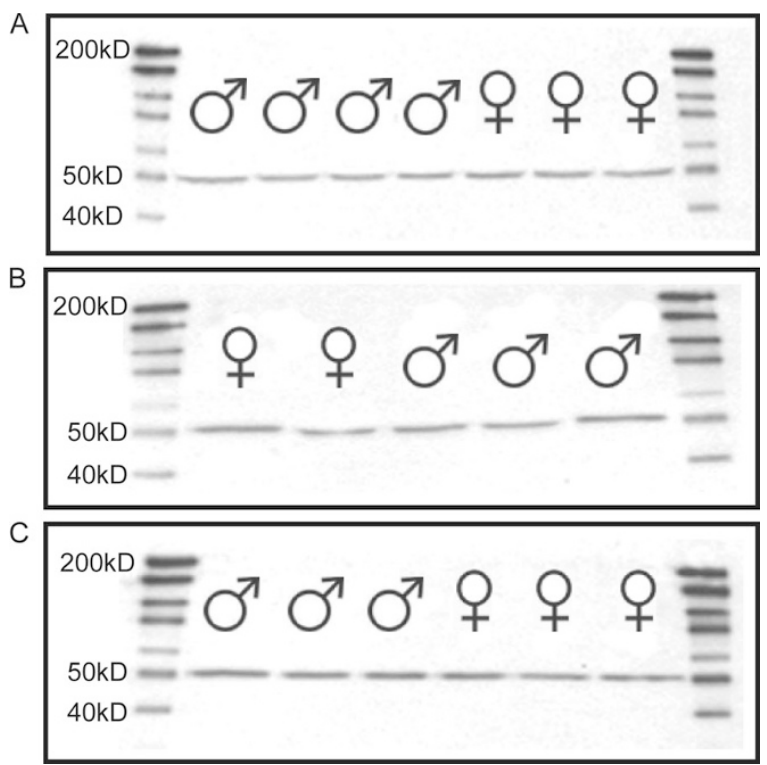

Figure 5. MOR is present centrally and peripherally on P0. After western blot analysis, MOR protein was present in the $(A)$ brain; $(B)$ spinal cord; $(C)$ hind paw on $\mathrm{P} 0$.

for the presence of MOR on maternal immune cells, we assessed the presence of MOR-LI on maternal blood samples taken at $5 \mathrm{~h}$ postparturition. MOR-LI was undetectable in maternal whole blood or serum (data not shown).

Neonatal injury has no impact on maternal care. There were no significant differences in maternal behavior between morphine pretreated and control animals in the amount of time the dam spent on/with pups (Fig. 6). Similarly, no differences were noted in the amount of time the dam spent off/without pups or in the amount of time spent licking/grooming pups (Fig. 6).

\section{DISCUSSION}

Our principal findings are as follows: 1) morphine pretreatment attenuates neonatal injury-induced hypoalgesia in adolescent and adult rats; 2) neonatal morphine reduces hyperalgesia after a subsequent inflammation in adulthood; 3) neonatal morphine increases the rate of recovery; 4) neonatal morphine does not affect morphine analgesia in adulthood; 5) neonatal injury significantly reduces morphine potency.

Neonatal morphine attenuates the consequences of neonatal injury. The present studies are the first to demonstrate that preemptive morphine attenuates the long-term behavioral consequences associated with neonatal intraplantar CGN in rodents. Morphine blocked thermal and mechanical hypoalgesia in the injured and uninjured paws in adolescence and adulthood in both sexes. This is consistent with previous studies in rodents that report daily morphine administration before intraplantar formalin during the first week of life significantly reduces the long-term effects of repetitive pain (22). Similarly, previous studies in humans have reported that morphine ameliorates the effects of early repetitive noxious stimuli in preterm infants at 4 mo of age (14). Moreover, children who had minor neonatal operations and received preemptive analgesia responded to immunization pain in a similar manner as controls (28).

There is ample evidence that the hypoalgesia observed after neonatal inflammation might be mediated by a potentiation in descending endogenous opioid tone. Studies have shown that the functional activity of endogenous opioid systems is enhanced after noxious inflammatory stimulation $(29,30)$. Furthermore, increased endogenous opioid peptide expression and release in the periaqueductal gray after peripheral inflammation has been reported, and is associated with decreased nociceptive sensitivity (31). Moreover, we have reported that systemic naloxone attenuates behavioral deficits in pain responsiveness associated with neonatal inflammation, suggesting that alterations in opioid tone may underlie the observed hypoalgesia (13). Our recent studies have demonstrated that injury-induced alterations in opioid tone primarily involve mu and delta opioid receptors in the periaqueductal gray (LaPrairie $\mathrm{J}$ 2007 SFN poster). These results are consistent with findings in former preterm infants $(32,33)$ and rodents treated neonatally with capsaicin (34) that early life trauma may impair the proper development of endogenous inhibitory systems in
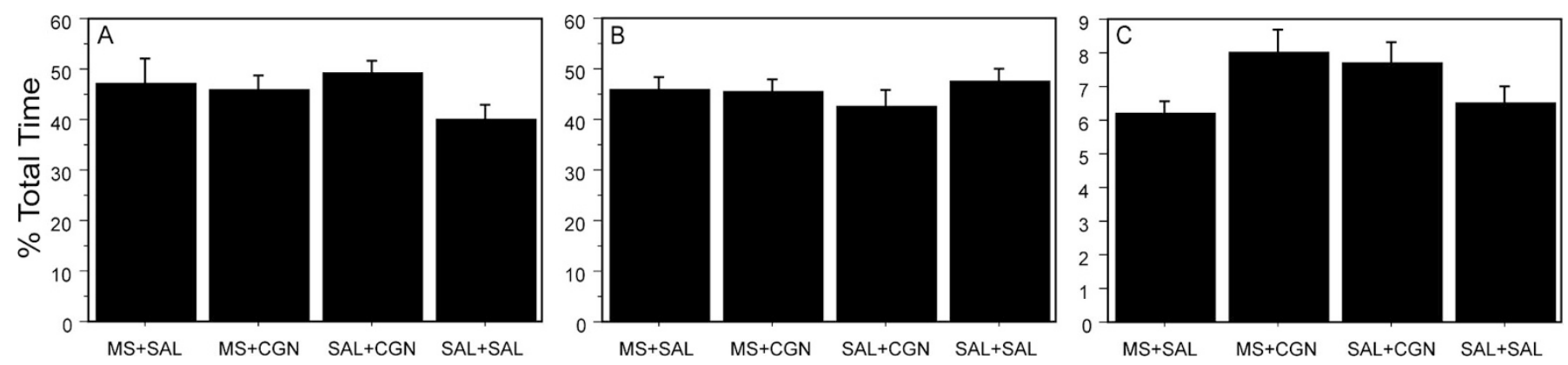

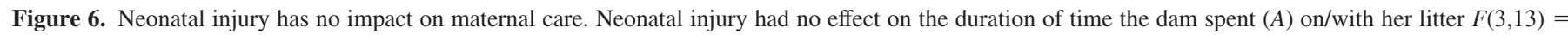

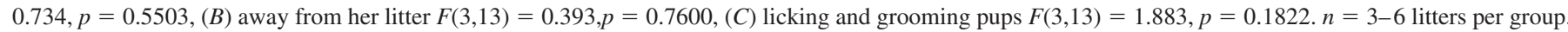


the CNS. In the present study, the ability of preemptive morphine to block the hypoalgesia may indeed occur through direct modulation of primary afferent drive into the spinal cord, thereby inhibiting the central relay of inflammatory pain and preventing the subsequent increase in descending endogenous opioid tone.

We cannot rule out alterations in the HPA axis as a contributing factor to the observed hypoalgesia. Nociception is one element in a broader context of stress reactivity $(35,36)$, and experimental studies have shown that exposure to early life stressors can permanently increase nociceptive thresholds and decrease the behavioral and physiologic responses to stress in adult rodents (36-38). Data from human preterm infants also suggest that neonatal exposure to noxious stimuli may alter future pain responses. Ex-preterm infants exposed to 4 wk of NICU care display reduced pain responsiveness after heel lance (39). In addition, preterm birth is associated with increased salivary cortisol in response to vaccination at $6 \mathrm{mo}$ of age (40).

Neonatal morphine reduces inflammatory hyperalgesia and increases the rate of recovery after re-injury. In the present study, morphine pretreatment significantly attenuated CFA-induced hyperalgesia and increased the rate of recovery, such that both males and females recovered $7 \mathrm{~d}$ faster than nonpretreated animals. Interestingly, preliminary anatomical studies in our laboratory suggest that neonatal inflammation results in increased primary afferent innervation of the dorsal horn reflected by increased expression of calcitonin generelated peptide (CGRP) and substance $\mathrm{P}$ immunoreactivity (LaPrairie JL, Murphy AZ, SfN abstract, 2005), which may account for our observed hyperalgesia. Our working hypothesis is that re-inflammation in adulthood results in an enhanced dorsal horn release of CGRP and/or substance P because of increased primary afferent input. Increased release of these pro-nociceptive peptides would be predicted to result in an enhanced hyperalgesic response. Clinical reports demonstrate that at $32 \mathrm{wk}$, preterm infants experience a reduced rate of recovery to skin breaking procedures (41), and exhibit subtle differences in ability to recover from finger lance at 4 mo compared with controls (15). There are no reports on the impact of preemptive morphine on recovery rates in premature neonates; however, our data suggest that morphine analgesia may in fact significantly increase the rate of recovery after procedural pain in NICU infants. Neonatal pretreatment with morphine may prevent the injury-induced increase in primary afferent innervation in the spinal cord, thereby blocking the susceptibility of re-inflammation to produce enhanced hyperalgesia and increasing the rate of recovery.

Neonatal morphine does not affect morphine analgesia in adulthood. Our original hypothesis was that exposure to morphine during neonatal development may alter opioid receptor number and/or affinity, thereby altering morphine's antinociceptive effects in adulthood. This hypothesis was based on previous studies that report a 60 -fold shift in the morphine dose-response cuve in adult rats that received repeated neonatal morphine in the absence of pain (17). Nevertheless, we show that neonatal morphine did not affect morphine analgesia in adult animals (i.e., no significant shift in
ED50 values). In contrast to the previous reports, however, neonatal morphine administration was associated with peripheral inflammation and was only provided on $\mathrm{P} 0$ in the present study.

Interestingly, neonatal injury alone resulted in a significant decrease in morphine potency in both males and females in adulthood. These results have serious clinical implications. Previous studies have reported that preterm infants that experience surgery during the first 3 mo of life have significantly higher peri- and postoperative analgesic requirements in response to surgery in the same or different dermatome compared with control infants $(21,40)$. Similarly, mice exposed to chronic noxious stimulation display increased TFLs compared with controls, and a significant 2-fold increase in the ED50 of morphine in response to abdominal constriction (42). As noxious stimulation during the neonatal period leads to increased activation of opioid systems in a manner analogous to the repeated application of exogenous opiates, these studies provide evidence that neonatal injury produces cross-tolerance to the analgesic effects of morphine thereby decreasing the subsequent effectiveness of morphine $(43,44)$. Again interestingly, exposure to morphine neonatally did not result in a significant shift in ED50 values. Therefore, we believe that opioid cross tolerance is associated with neonatal injuryinduced chronic exposure to endogenous opioids resulting from a potentiation of the descending inhibitory circuit, and not a result of exposure to morphine on P0. Alternatively, neonatal stress associated with maternal separation and repeated handling is also associated with reduced opioid analgesia $(36,37)$. This suggests that alterations in opioid analgesia may reflect a combined effect of neonatal nociceptive experience as well as early life stress, and therefore may involve altered responsiveness of endogenous analgesia circuits and the hypothalamic-pituitary-adrenal axis.

\section{REFERENCES}

1. Tibboel D, Anand KJ, van den Anker JN 2005 The pharmacological treatment of neonatal pain. Semin Fetal Neonatal Med 10:195-205

2. Andrews K, Fitzgerald M 1999 Cutaneous flexion reflex in human neonates: a quantitative study of threshold and stimulus-response characteristics after single and repeated stimuli. Dev Med Child Neurol 41:696-703

3. Smith RP, Gitau R, Glover V, Fisk NM 2000 Pain and stress in the human fetus. Eur J Obstet Gynecol Reprod Biol 92:161-165

4. Giannakoulopoulos X, Teixeira J, Fisk N, Glover V 1999 Human fetal and maternal noradrenaline responses to invasive procedures. Pediatr Res 45:494-499

5. Anand KJ, Carr DB 1989 The neuroanatomy, neurophysiology, and neurochemistry of pain, stress, and analgesia in newborns and children. Pediatr Clin North Am 36:795-822

6. Franck LS, Greenberg CS, Stevens B 2000 Pain assessment in infants and children. Pediatr Clin North Am 47:487-512

7. McNair C, Ballantyne M, Dionne K, Stephens D, Stevens B 2004 Postoperative pain assessment in the neonatal intensive care unit. Arch Dis Child Fetal Neonatal Ed 89:F537-F541

8. Slater R, Cantarella A, Gallella S, Worley A, Boyd S, Meek J, Fitzgerald M 2006 Cortical pain responses in human infants. J Neurosci 26:3662-3666

9. Bartocci M, Bergqvist LL, Lagercrantz H, Anand KJ 2006 Pain activates cortical areas in the preterm newborn brain. Pain 122:109-117

10. Grunau RV, Whitfield MF, Petrie JH 1994 Pain sensitivity and temperament in extremely low-birth-weight premature toddlers and preterm and full-term controls. Pain 58:341-346

11. Bhutta AT, Cleves MA, Casey PH, Cradock MM, Anand KJ 2002 Cognitive and behavioral outcomes of school-aged children who were born preterm: a metaanalysis. JAMA 288:728-737

12. Lidow MS, Song ZM, Ren K 2001 Long-term effects of short-lasting early local inflammatory insult. Neuroreport 12:399-403

13. LaPrairie JL, Murphy AZ 2007 Female rats are more vulnerable to the long-term consequences of neonatal inflammatory injury. Pain 132:S124-S133 
14. Grunau RE, Oberlander TF, Whitfield MF, Fitzgerald C, Lee SK 2001 Demographic and therapeutic determinants of pain reactivity in very low birth weight neonates at 32 Weeks' postconceptional Age. Pediatrics 107:105-112

15. Oberlander TF, Grunau RE, Whitfield MF, Fitzgerald C, Pitfield S, Saul JP 2000 Biobehavioral pain responses in former extremely low birth weight infants at four months' corrected age. Pediatrics 105:e6

16. Wang G, Ji Y, Lidow MS, Traub RJ 2004 Neonatal hind paw injury alters processing of visceral and somatic nociceptive stimuli in the adult rat. J Pain 5:440-449

17. Bhutta AT, Rovnaghi C, Simpson PM, Gossett JM, Scalzo FM, Anand KJ 2001 Interactions of inflammatory pain and morphine in infant rats: long-term behavioral effects. Physiol Behav 73:51-58

18. Anand KJ, Hickey PR 1992 Halothane-morphine compared with high-dose sufentanil for anesthesia and postoperative analgesia in neonatal cardiac surgery. $\mathrm{N}$ Engl J Med 326:1-9

19. Yaster M, Deshpande JK 1988 Management of pediatric pain with opioid analgesics. J Pediatr 113:421-429

20. Bouwmeester NJ, Anand KJ, van Dijk M, Hop WC, Boomsma F, Tibboel D 2001 Hormonal and metabolic stress responses after major surgery in children aged 0-3 years: a double-blind, randomized trial comparing the effects of continuous versus intermittent morphine. Br J Anaesth 87:390-399

21. Bouwmeester NJ, Hop WC, van Dijk M, Anand KJ, van den Anker JN, Tibboel D 2003 Postoperative pain in the neonate: age-related differences in morphine requirements and metabolism. Intensive Care Med 29:2009-2015

22. Abbott FV, Guy ER 1995 Effects of morphine, pentobarbital and amphetamine on formalin-induced behaviours in infant rats: sedation versus specific suppression of pain. Pain 62:303-312

23. Sternberg WF, Scorr L, Smith LD, Ridgway CG, Stout M 2005 Long-term effects of neonatal surgery on adulthood pain behavior. Pain 113:347-353

24. Nandi R, Beacham D, Middleton J, Koltzenburg M, Howard RF, Fitzgerald M 2004 The functional expression of mu opioid receptors on sensory neurons is developmentally regulated; morphine analgesia is less selective in the neonate. Pain $111: 38-50$

25. Hargreaves K, Dubner R, Brown F, Flores C, Joris J 1988 A new and sensitive method for measuring thermal nociception in cutaneous hyperalgesia. Pain 32:77-88

26. Morgan MM, Fossum EN, Stalding BM, King MM 2006 Morphine antinociceptive potency on chemical, mechanical, and thermal nociceptive tests in the rat. J Pain 7:358-366

27. Wang X, Traub RJ, Murphy AZ 2006 Persistent pain model reveals sex difference in morphine potency. Am J Physiol Regul Integr Comp Physiol 291:R300-R306

28. Peters JW, Koot HM, de Boer JB, Passchier J, Bueno-de-Mesquita JM, de Jong FH, Duivenvoorden HJ, Tibboel D 2003 Major surgery within the first 3 months of life and subsequent biobehavioral pain responses to immunization at later age: a case comparison study. Pediatrics 111:129-135

29. Iadarola MJ, Douglass J, Civelli O, Naranjo JR 1988 Differential activation of spinal cord dynorphin and enkephalin neurons during hyperalgesia: evidence using cDNA hybridization. Brain Res 455:205-212
30. Noguchi K, Morita Y, Kiyama H, Sato M, Ono K, Tohyama M 1989 Preproenkephalin gene expression in the rat spinal cord after noxious stimuli. Brain Res Mol Brain Res 5:227-234

31. Williams FG, Mullet MA, Beitz AJ 1995 Basal release of Met-enkephalin and neurotensin in the ventrolateral periaqueductal gray matter of the rat: a microdialysis study of antinociceptive circuits. Brain Res 690:207-216

32. Goffaux P, Lafrenaye S, Morin M, Patural H, Demers G, Marchand S. Preterm births: can neonatal pain alter the development of endogenous gating systems? Eur $\mathrm{J}$ Pain, in press

33. Hermann C, Hohmeister J, Demirakca S, Zohsel K, Flor H 2006 Long-term alteration of pain sensitivity in school-aged children with early pain experiences. Pain 125:278-285

34. Cervero F, Plenderleith MB 1985 C-fibre excitation and tonic descending inhibition of dorsal horn neurones in adult rats treated at birth with capsaicin. J Physiol 365:223-237

35. Grunau RE, Holsti L, Haley DW, Oberlander T, Weinberg J, Solimano A, Whitfield MF, Fitzgerald C, Yu W 2005 Neonatal procedural pain exposure predicts lower cortisol and behavioral reactivity in preterm infants in the NICU. Pain 113:293-300

36. Sternberg WF, Ridgway CG 2003 Effects of gestational stress and neonatal handling on pain, analgesia, and stress behavior of adult mice. Physiol Behav 78:375-383

37. Coutinho SV, Plotsky PM, Sablad M, Miller JC, Zhou H, Bayati AI, McRoberts JA, Mayer EA 2002 Neonatal maternal separation alters stress-induced responses to viscerosomatic nociceptive stimuli in rat. Am J Physiol Gastrointest Liver Physiol 282:G307-G316

38. Pieretti S, d'Amore A, Loizzo A 1991 Long-term changes induced by developmental handling on pain threshold: effects of morphine and naloxone. Behav Neurosci 105:215-218

39. Johnston CC, Stevens BJ 1996 Experience in a neonatal intensive care unit affects pain response. Pediatrics 98:925-930

40. Peters JW, Schouw R, Anand KJ, van Dijk M, Duivenvoorden HJ, Tibboel D 2005 Does neonatal surgery lead to increased pain sensitivity in later childhood? Pain 114:444-454

41. Morison SJ, Holsti L, Grunau RE, Whitfield MF, Oberlander TF, Chan HW, Williams L 2003 Are there developmentally distinct motor indicators of pain in preterm infants? Early Hum Dev 72:131-146

42. Christie MJ, Trisdikoon P, Chesher GB 1982 Tolerance and cross tolerance with morphine resulting from physiological release of endogenous opiates. Life Sci 31:839-845

43. Lett BT, Grant VL, Koh MT, Flynn G 2002 Prior experience with wheel running produces cross-tolerance to the rewarding effect of morphine. Pharmacol Biochem Behav 72:101-105

44. Stoller DC, Thornton SR, Smith FL 2002 Loss of antinociceptive efficacy in rat pups infused with morphine from osmotic minipumps. Pharmacology 66:11-18 